\title{
MicroRNA-494 promotes cervical cancer proliferation through the regulation of PTEN
}

\author{
YONG-KANG YANG ${ }^{1}$, WEN-YAN XI ${ }^{1}$, RU-XING XI $^{2}$, JING-YUAN LI $^{3}$, QIN LI $^{4}$ and YAN-E GAO ${ }^{1}$ \\ ${ }^{1}$ Department of Gynecology and Obstetrics, The Second Affiliated Hospital of Medical School of Xi'an Jiaotong University, \\ Xi'an, Shaanxi 710004; ${ }^{2}$ Department of Radiotherapy, The First Affiliated Hospital of Medical College of Xi'an Jiaotong \\ University, Xi'an, Shaanxi 710061; ${ }^{3}$ Department of Orthopedics, The Second Affiliated Hospital of Medical School \\ of Xi'an Jiaotong University, Xi'an, Shaanxi 710004; ${ }^{4}$ Department of Gynecology and Obstetrics, The Second \\ Affiliated Hospital of Shaanxi University of Chinese Medicine, Xianyang, Shaanxi 712000, P.R. China
}

Received December 1, 2014; Accepted February 2, 2015

DOI: $10.3892 /$ or.2015.3821

\begin{abstract}
The phosphoinositide 3-kinase (PI3K)/Akt signaling pathway appears to be a key regulator in cervical carcinogenesis. The phosphatase and tensin homolog deleted on chromosome 10 (PTEN) protein is principally involved in the homeostatic maintenance of PI3K/Akt signaling and PTEN has been identified to play an important role in the occurrence and development of cervical cancer. MicroRNA (miRNA)-494 has been proven to be involved in the carcinogenesis and development of various types of cancer by directly targeting PTEN. However the role, mechanism and clinical significance of miR-494 in cervical cancer have not been further reported. In the present study, we analyzed the expression of miR-494 in cervical cancer cell lines and clinical specimens by RT-qPCR, and explored the association of miR-494 with PTEN expression and clinicopathological data of cervical cancer patients. The results showed that miR-494 expression was significantly upregulated in human cervical cancer cell lines and tissues. miR-494 upregulation was significantly associated with PTEN downregulation, adverse clinicopathological characteristics, poor overall and progression-free survival and poor prognosis. In vitro experiments showed that inhibition of miR-494 suppressed cell proliferation and growth by directly targeting
\end{abstract}

Correspondence to: Professor Yan-E Gao, Department of Gynecology and Obstetrics, The Second Affiliated Hospital of Medical School of Xi'an Jiaotong University, 157 Xiwu Road, Xi'an, Shaanxi 710004, P.R. China

E-mail: yanegao@163.com

Abbreviations: miR-494, microRNA-494; HR-HPV, high-risk human papilloma virus; PTEN, phosphatase and tensin homolog deleted on chromosome 10; NCEC, normal cervical epithelial cells; WT, wild-type; MT, mutant; 3'-UTR, 3'-untranslated region; RT-qPCR, reverse transcription-quantitative polymerase chain reaction; MMAC1, mutated in multiple advanced cancer 1

Key words: miR-494, cervical cancer, proliferation, PI3K/Akt, PTEN the 3'-untranslated region (3'-UTR) of PTEN mRNA. These findings identified a novel molecular mechanism involved in the regulation of PTEN expression and cervical cancer progression. Results of the present study indicated that miR-494 may have an essential role in the carcinogenesis and progression of cervical cancer and targeting miR-494 may be a promising therapeutic strategy for the treatment of cervical cancer.

\section{Introduction}

Cervical cancer is the fourth most prevalent cause of cancer-related mortality in women worldwide and 12,360 estimated new cases of cervical cancer were diagnosed in 2014, with 4,020 estimated deaths in the USA (1). Significant advances concerning the molecular mechanisms of cervical carcinogenesis have been made during the last several decades (2). However, the detailed mechanisms of cervical cancer initiation and progression have yet to be fully elucidated. Persistent infection with high-risk human papilloma virus (HR-HPV) has been proven to be the main cause of almost all types of cervical cancer. However, a substantial body of evidence shows that HR-HPV infection alone is not sufficient to induce malignant transformation, indicating that other genetic alterations may be involved in cervical carcinogenesis (3). Identification of key factors in cervical cancer is important for the screening, diagnosis and treatment of cervical cancer.

The phosphoinositide 3-kinase (PI3K)/Akt signaling pathway appears to be a key regulator in cervical carcinogenesis, as it is activated in $>90 \%$ of cervical cancer types (4). Akt signaling is the downstream target of HPV oncoproteins which have been identified as major mediators of cervical cancer initiation and development (5). Gene expression profiling also demonstrated that the PI3K/Akt signaling pathway may be of potential therapeutic target in cervical cancer (6). The phosphatase and tensin homolog deleted on chromosome 10 (PTEN) protein is principally involved in the homeostatic maintenance of the PI3K/Akt signaling pathway (7). Findings of a previous study showed that loss of PTEN resulted in persistent activation of PI3K effectors which has an important impact on various aspects of cancer development such as cell proliferation, cell cycle, cell migration and metastasis (8). It 
has been demonstrated that abnormal promoter methylation of the PTEN gene was usually identified in cervical cancer and associated with tumor differentiation, lymph-node metastasis and FIGO staging. PTEN was identified to be important in the occurrence and development of cervical cancer (9). Therefore, identification of the molecular regulating PTEN expression may be an attractive strategy for elucidating the underlying mechanism of cervical carcinogenesis.

MicroRNAs (miRNAs) are small non-coding RNAs of 22 nucleotides in length, transcribed from non-protein coding genes or introns, which generally act as negative regulators of gene expression at post-transcriptional levels through mRNA degradation and translation repression (10). Accumulating evidence has shown that the aberrant expression of miRNAs may function as tumor suppressors or oncogenes in cancers according to the role of their target genes (11), which indicates miRNAs have the potential to be diagnostic and prognostic biomarkers for cancer (12). miR-494 has consistently been reported to be aberrantly expressed in various types of cancer. The functional role of miR-494 is extremely complex as it may function as an oncogenic or tumor suppressive miRNA depending on the cellular microenvironments (13-17). More importantly, it has been reported that miR-494 participated in the carcinogenesis and development of colorectal cancers by directly targeting PTEN (18). Aberrant miRNA expression profiles have also been identified in cervical cancer cell lines and cervical cancer tissues $(19,20)$. However, to the best of our knowledge, there are few detailed studies focusing on the role of miR-494 in cervical cancer. Given the complexity of its functionality, it would be of interest to explore the functional roles and relationship of miR-494 and PTEN in cervical cancer carcinogenesis and development.

In the present study, we analyzed the expression of miR-494 in cervical cancer cell lines and clinical specimens, and examined the association of miR-494 with PTEN expression and clinicopathological data of cervical cancer patients. In vitro experiments showed that inhibition of miR-494 suppressed cell proliferation and growth by directly targeting the 3'-untranslated region (3'-UTR) of PTEN mRNA. These findings identified a novel molecular mechanism involved in the regulation of PTEN expression and cervical cancer progression. Thus, targeting miR-494 may be a promising therapeutic strategy for the treatment of cervical cancer.

\section{Materials and methods}

Patients and tissue specimens. The tissue-based specimen collection and study were approved by the Research Ethics Committee of Xi'an Jiaotong University. All the patients provided written consent and indicated willingness to donate their blood and tissue samples. A total of 89 patients were enrolled in the present study. Clinical and pathological classification and staging were performed according to the International Federation of Gynecology and Obstetrics criteria (21). The clinicopathological information of the patients is shown in Table I. The follow-up information for all participants was updated every 3 months by telephone. Information regarding the death of patients was ascertained from their family. In all 89 snap-frozen cervical cancer samples, the HC2 assay was used to detect the presence of high-risk HPV DNA, including DNA from HPV types 16, 18, 31, 33, 35, 39, 45, 51, 52, 56, 58, 59 and 68 (22). High-risk HPV (HR-HPV) was detected in 79 cases, which gave an overall infection rate of $88.8 \%$.

Cell culture. Primary normal cervical epithelial cells (NCEC) obtained from healthy female cervical tissue were cultured in keratinocyte serum-free medium (Invitrogen, Carlsbad, CA, USA) supplemented with epithelial growth factor, bovine pituitary extract and antibiotics (1\% streptomycin and 1\% penicillin). The HeLa, C33A, Caski and SiHa cervical cancer cell lines were grown in Dulbecco's modified Eagle's medium (DMEM) (Invitrogen). The cells were supplemented with $10 \%$ fetal bovine serum (FBS) (HyClone, Logan, UT, USA) and 1\% penicillin/streptomycin (Invitrogen).

Reverse transcriptase-quantitative PCR (RT-qPCR) assay. The expression of miR-494 in cervical cancer and corresponding adjacent tissues was detected by the RT-qPCR assay. Briefly, total RNA was extracted from tissues using TRIzol reagent (Invitrogen) according to the manufacturer's instructions. miRNA expression levels were quantified using a TaqMan miRNA real-time RT-PCR kit (Applied Biosystems, Foster City, CA, USA) according to the manufacturer's instructions. Data were analyzed using 7500 software v. 2.0.1 (Applied Biosystems), with the automatic Ct setting for adapting the baseline and threshold for $\mathrm{Ct}$ determination. The universal small nuclear RNA U6 (RNU6B) was used as an endogenous control for miRNAs. Each sample was examined in triplicate and the amount of PCR products produced was non-neoplasticized to RNU6B.

Oligonucleotide transfection. miR-494 inhibitors were chemically synthesized by Shanghai GenePharma (GenePharma, Shanghai, China). When the cells reached $80 \%$ confluence, miR-494 inhibitor was transfected into cervical cancer cells with Lipofectamine 2000 (Invitrogen) according to the manufacturer's instructions. The cells were also transfected with scramble oligonucleotide as a negative control (NC). The expression level of mir-494 in the transfected osteosarcoma cells were identified by RT-qPCR.

Luciferase reporter assay. Cervical cancer cells were seeded in 96-well plates at $60 \%$ confluence. After $24 \mathrm{~h}$, the cells were transfected with $120 \mathrm{ng}$ of miR-494 expression vector or NC. The cells were transfected with $30 \mathrm{ng}$ of wild-type (WT) or mutant (MT) 3'-UTR of PTEN mRNA. The cells were collected $48 \mathrm{~h}$ after transfection, and luciferase activity was measured using a dual luciferase reporter assay system according to the manufacturer's instructions (Promega, Madison, WI, USA).

Cell viability assay. Cells were plated in 96-well plates $\left(0.5 \times 10^{4}\right.$ cells/well $)$ and transfected with $\mathrm{NC}$, and miR-494 inhibitors. After $48 \mathrm{~h}, 10 \mu \mathrm{l}$ of MTT reagent $(5 \mathrm{mg} / \mathrm{ml})$ was added to each well and the cells were incubated at $37^{\circ} \mathrm{C}$ for another $4 \mathrm{~h}$. The medium was removed, the cells were solubilized in $150 \mu$ l of dimethyl sulfoxide, and colorimetric analysis was performed (wavelength, $490 \mathrm{~nm}$ ). One plate was analyzed immediately after the cells adhered ( $4 \mathrm{~h}$ after plating), and the remaining plates were assayed every day for the following 4 consecutive days. 
A

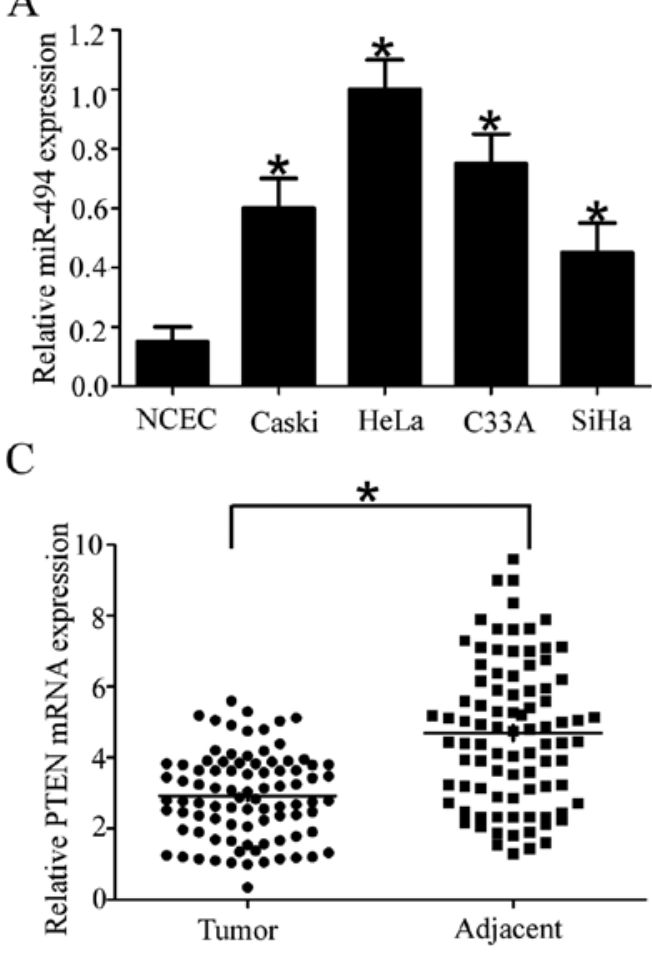

$\mathrm{B}$

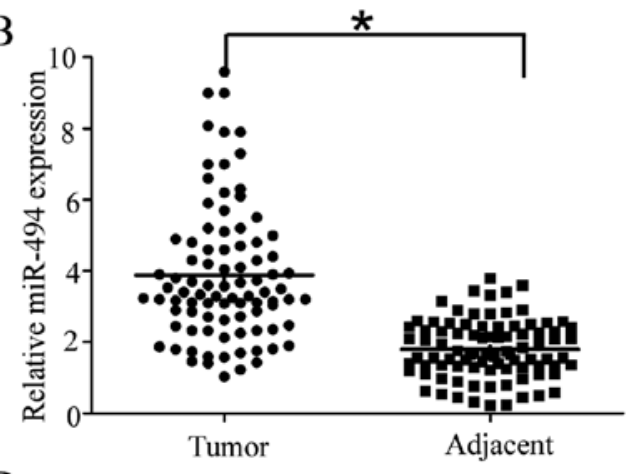

D

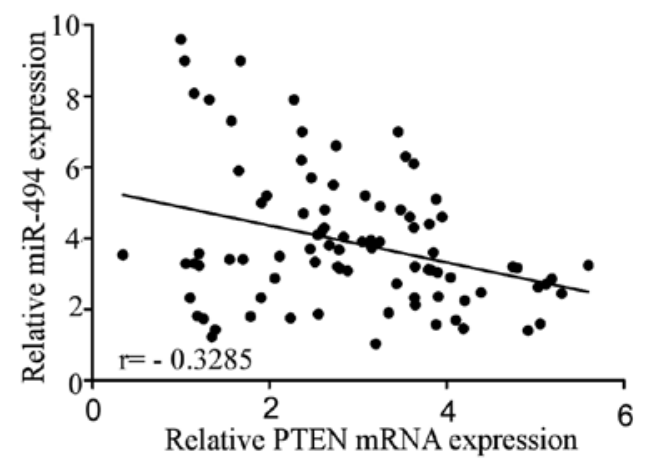

Figure 1. Expression of miR-494 and PTEN is increased in cervical cancer cell lines and tissues. (A) Expression of miR-494 in normal cervical epithelial cells (NCEC) and cervical cancer cell lines (Caski, HeLa, C33A and SiHa). (B) Expression of miR-494 in 89 paired cervical cancer tissues and their adjacent normal tissues. (C) Expression of PTEN mRNA in 89 paired cervical cancer and their adjacent normal tissues. (D) Correlation between miR-494 and PTEN mRNA levels in cervical cancer tissues. Expression levels of miR-494 and PTEN were determined by RT-qPCR and normalized against an endogenous control U6 RNA and $\beta$-actin, respectively. ${ }^{*} \mathrm{P}<0.05$. PTEN, phosphatase and tensin homolog deleted on chromosome 10.

Colony formation assay. Briefly, $10 \mathrm{~cm}$ dishes were seeded with 500 viable cells in complete medium and allowed to grow for $24 \mathrm{~h}$. The cells were then incubated in the presence of miR-494 inhibitors or NC for up to $48 \mathrm{~h}$. The medium was removed, and the cells were washed in phosphate-buffered saline (PBS) and incubated for an additional 10 days in complete medium. Each treatment was carried out in triplicate. The colonies obtained were washed with PBS and fixed in $4 \%$ formalin for $10 \mathrm{~min}$ at room temperature and then washed with PBS followed by staining with $0.2 \%$ crystal violet.

Soft agar colony formation assay. Cells seeded in a 6-well plate were covered with a layer of $0.6 \%$ agar in DMEM medium supplemented with $10 \%$ FBS. After transfection for $48 \mathrm{~h}$, the cells were trypsinized, gently mixed with $0.3 \%$ agar medium mixture containing selective antibiotics and reseeded in triplicate in a 6-well plate. After 4 weeks, the resistant colonies were stained with $0.2 \%$ crystal violet and counted under the microscope.

Flow cytometric analysis of cell cycle. The cervical cancer cells were transfected with NC and miR-101 inhibitors. Forty-eight hours after post-transfection, the cells were trypsinized and analyzed for cell cycle distribution. For cell cycle distribution, the cells of each group were stained with propidium iodide (PI) and analyzed by flow cytometry using FACSCalibur (BD Biosciences, San Diego, CA, USA). For each group, 10,000 events were obtained. The percentage of cells in G1, S and G2 phases of the cell cycle was calculated.
Statistical analysis. Data are presented as mean \pm SD. Statistical analysis was performed using IBM SPSS statistical software (version 21.0) (International Business Machines Corporation, Armonk, NY, USA). The differences in characteristics between the two groups were examined by the $\chi^{2}$ or Fisher's exact tests. $\mathrm{P}$-values were determined from two-sided tests, and statistical significance was based on a P-value of 0.05 .

\section{Results}

miR-494 is upregulated in cervical cancer cell lines and tissues. To examine the levels of miR-494 expression in cervical cancer, we conducted RT-qPCR to measure miR-494 expression in four cervical cancer cell lines and NCEC. The result showed that miR-494 was markedly increased in the Caski, HeLa, C33A and SiHa cervical cell lines, particularly in HeLa and C33A, compared with NCEC (Fig. 1A). Consistent with the results found in cervical cell lines, miR-494 expression was significantly higher in 89 cervical cancer tissue specimens compared with their adjacent normal tissues (Fig. 1B). By contrast, the expression of PTEN was significantly downregulated in cervical cancer tissues compared with their normal tissue counterparts (Fig. 1C), which was consistent with previous literature (23). More importantly, statistically significant inverse correlations were revealed by Spearman's correlation analysis between mRNA levels of miR-494 and PTEN in cervical cancer specimens $(r=-0.3285 ; \mathrm{P}=0.0017)$. Taken together, the results suggested that miR-494 played an oncogenic role and PTEN a tumor-suppressor role in cervical 

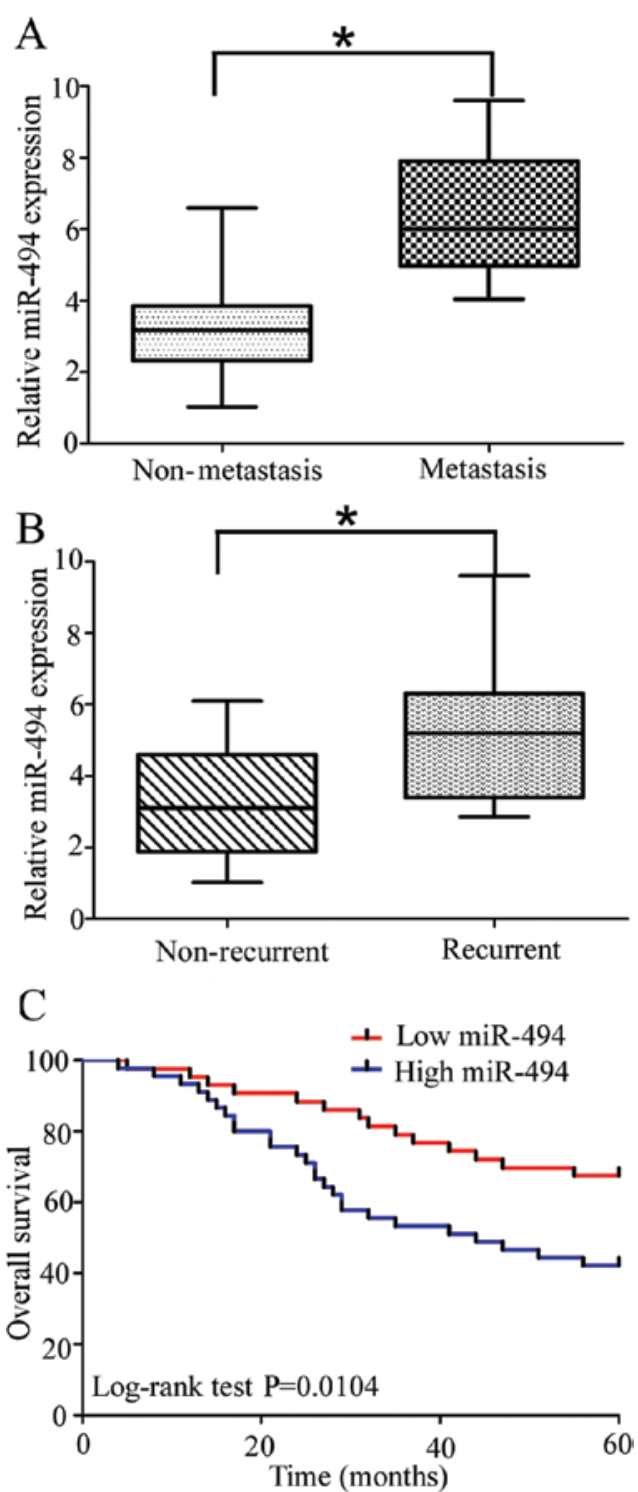

D

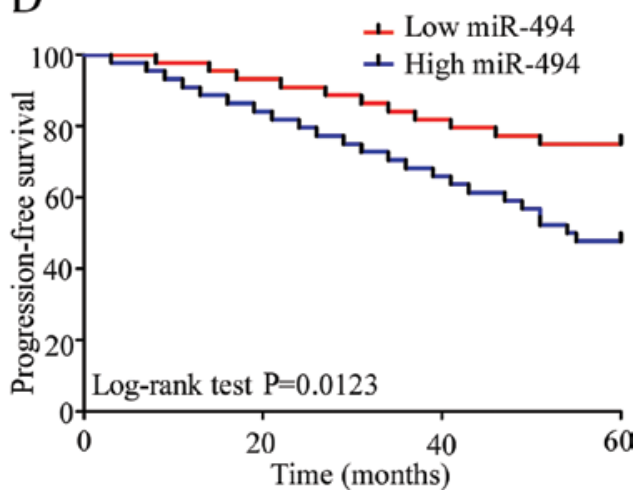

Figure 2. Clinical significance of miR-494 in cervical cancer patients. (A) Comparison of miR-494 levels between metastatic and non-metastatic cervical cancer patients. (B) Comparison of miR-494 levels between recurrent and non-recurrent cervical cancer patients. (C) Overall survival curves for low and high expression of miR-494 in cervical cancer patients. (D) Progression-free survival curves for low and high expression of miR-494 in cervical cancer patients. Expression levels of miR-494 were determined by RT-qPCR and normalized against an endogenous control U6 RNA. "P<0.05.

cancer. Furthermore, miR-494 inversely correlated with PTEN in cervical cancer, which indicated that PTEN was a potential target of miR-494 in cervical cancer.
Table I. Association between miR-494 expression and clinicopathological characteristics.

\begin{tabular}{|c|c|c|c|c|}
\hline \multirow[b]{2}{*}{ Characteristics } & \multirow[b]{2}{*}{ No. } & \multicolumn{2}{|c|}{$\begin{array}{c}\text { miR-494 } \\
\text { expression }\end{array}$} & \multirow[b]{2}{*}{ P-value } \\
\hline & & Low & High & \\
\hline Age (years) & & & & 0.666 \\
\hline$\leq 35$ & 35 & 16 & 19 & \\
\hline$>35$ & 54 & 28 & 26 & \\
\hline FIGO stage & & & & $0.026^{\mathrm{a}}$ \\
\hline IB & 58 & 34 & 24 & \\
\hline$>\mathrm{IB}$ & 31 & 10 & 21 & \\
\hline HR-HPV & & & & 0.091 \\
\hline Yes & 79 & 38 & 41 & \\
\hline No & 10 & 8 & 2 & \\
\hline Differentiation & & & & 0.833 \\
\hline Well & 41 & 21 & 20 & \\
\hline Moderate/poor & 48 & 23 & 25 & \\
\hline Tumor size & & & & 0.477 \\
\hline$\leq 4 \mathrm{~cm}$ & 70 & 33 & 37 & \\
\hline$>4 \mathrm{~cm}$ & 19 & 11 & 8 & \\
\hline LN metastasis & & & & $0.027^{\mathrm{a}}$ \\
\hline Yes & 78 & 35 & 43 & \\
\hline No & 11 & 9 & 2 & \\
\hline Stromal invasion & & & & $0.045^{\mathrm{a}}$ \\
\hline$<2 / 3$ & 68 & 38 & 30 & \\
\hline$\geq 2 / 3$ & 21 & 6 & 15 & \\
\hline
\end{tabular}

aSignificant difference of clinical factors with overall survival. HR-HPV, high-risk human papilloma virus.

Upregulation of miR-494 is associated with metastasis and recurrence in cervical cancer patients. To explore the relationship between miR-494 and cervical cancer, we investigated the correlation of miR-494 expression with metastasis and recurrence of cervical cancer. Compared with non-metastatic cervical cancer specimens, the miR-494 levels were significantly upregulated in metastatic cervical tissues (Fig. 2A). Moreover, miR-494 levels were significantly higher in the specimens obtained from the patients who suffered cervical cancer recurrence (Fig. 1B). Collectively, these data indicated that significantly upregulation of miR-494 expression was correlated with relapse and metastasis in cervical cancer patents.

miR-494 expression is correlated with clinicopathological characteristics and prognosis in cervical cancer patients. In order to determine the clinical significance of miR-494 in cervical cancer, the 89 patients were divided into two groups based on miR-494 expression levels (low vs. high) with the median expression levels as a cut-off point. The Kaplan-Meier analysis revealed that high miR-494 expression was significantly correlated with reduced overall and progression-free survival in 89 cervical cancer patients (Fig. 2C and D; log-rank test, 
Table II. Univariate and multivariate analyses of clinical parameters in relation to overall survival.

\begin{tabular}{lcccc}
\hline & \multicolumn{2}{c}{ Univariate } & & Multivariate \\
\cline { 2 - 4 } Variables & HR $(95 \% \mathrm{CI})$ & P-value & HR $(95 \% \mathrm{CI})$ \\
\hline miR-494 expression & $4.143(1.751-6.397)$ & $0.009^{\mathrm{a}}$ & $3.279(1.177-5.192)$ & $0.013^{\mathrm{a}}$ \\
Age (years) & $2.145(0.745-2.451)$ & 0.658 & $1.784(0.874-2.175)$ & 0.791 \\
FIGO stage & $4.156(2.209-5.167)$ & $0.017^{\mathrm{a}}$ & $3.516(2.124-5.349)$ & $0.009^{\mathrm{a}}$ \\
HR-HPV & $3.129(1.296-4.719)$ & $0.045^{\mathrm{a}}$ & $2.891(1.152-4.325)$ & $0.021^{\mathrm{a}}$ \\
Differentiation & $1.819(0.742-2.795)$ & 0.209 & $2.113(0.696-2.782)$ & 0.491 \\
Tumor size & $1.361(0.534-1.987)$ & 0.419 & $0.542(0.759-2.175)$ & $0.008^{\mathrm{a}}$ \\
LN metastasis & $3.714(1.892-5.562)$ & $0.017^{\mathrm{a}}$ & & $0.115(1.579-6.123)$ \\
Stromal invasion & $1.193(0.415-1.987)$ & 0.118 & $1.453(0.879-2.161)$ \\
\hline
\end{tabular}

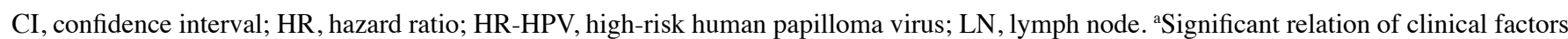
with overall survival.

Table III. Univariate and multivariate analyses of clinical parameters in relation to progression-free survival.

\begin{tabular}{|c|c|c|c|c|}
\hline \multirow[b]{2}{*}{ Variables } & \multicolumn{2}{|c|}{ Univariate } & \multicolumn{2}{|c|}{ Multivariate } \\
\hline & $\operatorname{HR}(95 \% \mathrm{CI})$ & P-value & $\mathrm{HR}(95 \% \mathrm{CI})$ & P-value \\
\hline miR-494 expression & $4.891(2.425-6.257)$ & $0.007 *$ & $4.614(2.895-10.321)$ & $<0.001^{\mathrm{a}}$ \\
\hline Age (years) & $1.427(0.628-2.162)$ & 0.351 & $1.891(0.898-2.477)$ & 0.519 \\
\hline FIGO stage & $3.451(1.679-4.129)$ & $0.029^{\mathrm{a}}$ & $2.915(1.789-4.187)$ & $0.011^{\mathrm{a}}$ \\
\hline HR-HPV & $3.198(1.589-5.245)$ & $0.014^{\mathrm{a}}$ & $2.941(1.497-4.827)$ & $0.008^{\mathrm{a}}$ \\
\hline Differentiation & $1.813(0.741-2.514)$ & 0.295 & $2.161(0.819-3.255)$ & 0.417 \\
\hline Tumor size & $1.429(0.711-2.287)$ & 0.342 & $1.827(0.717-3.165)$ & 0.417 \\
\hline $\mathrm{LN}$ metastasis & $5.104(1.998-10.179)$ & $0.029^{\mathrm{a}}$ & $4.219(2.326-7.619)$ & $0.031^{\mathrm{a}}$ \\
\hline Stromal invasion & $1.355(0.611-2.341)$ & 0.173 & $1.625(0.681-2.749)$ & $0.251^{\mathrm{a}}$ \\
\hline
\end{tabular}

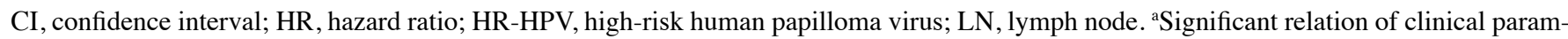
eters with progression-free survival.

$\mathrm{P}=0.0104$ and $\mathrm{P}=0.0123$, respectively). The patients with a high miR-494 expression tended to have a shorter overall and progression-free survival time when compared to patients with a low miR-494 expression. In addition, upregulation of miR-494 was significantly correlated with FIGO stage, lymph-node metastasis and deep stromal invasion while no significant correlation was observed in other clinicopathological variables (Table I). The, univariate analysis demonstrated that the overall and progression-free survival of cervical cancer patients was associated with FIGO stage, lymph-node status, and HR-HPV and miR-494 expression (Tables II and III). To determine whether the prognostic value of miR-494 was independent of other clinicopathological parameters for poor overall and progression-free survival in cervical cancer patients, a multivariate analysis was performed using a Cox proportional hazard model. The multivariate analysis including miR-494 expression, age, FIGO stage, HR-HPV, differentiation status, tumor size and lymph-node metastasis demonstrated that a high miR-494 expression was an independent prognostic biomarker for poor overall and progression-free survival in cervical cancer patients (Tables II and III; HR=3.279, $\mathrm{CI}=1.177-5.192, \mathrm{P}=0.013$ and $\mathrm{HR}=4.614, \mathrm{CI}=2.895-10.321$, $\mathrm{P}<0.001$ respectively). Statistically significant results were also obtained for FIGO stage and lymph-node metastasis, where the other parameters were not independent prognostic biomarkers for overall and progression-free survival in cervical cancer patients. Taken together, these results suggest the upregulation of miR-494 was significantly correlated with a worse prognosis and was involved in the progression of cervical cancer.

miR-494 promotes the proliferation of cervical cancer cells. As the relative expression of miR-494 was relatively higher in HeLa and C33A than SiHa and Caski, we chose HeLa and C33A to investigate the physiological function of miR-494 in cervical cancer cells. To analyze the effect of miR-494 on the proliferation of cervical cancer cells, we transfected miR-494 inhibitors into HeLa and C33A cell lines. As shown in Fig. 3A, transfection of miR-494 inhibitors decreased the miR-494 expression 
A

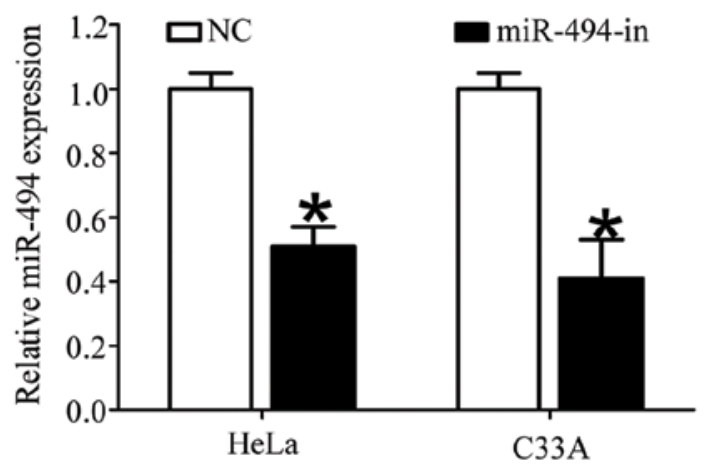

B

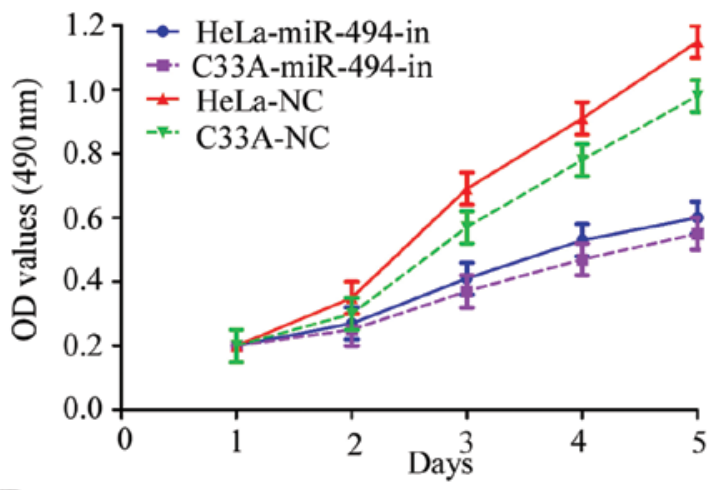

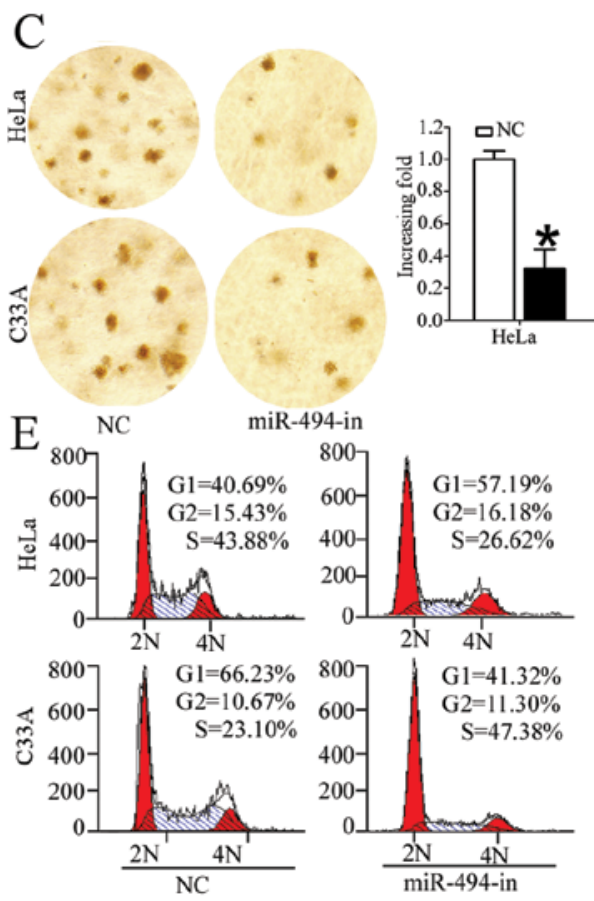

$\mathrm{D}$
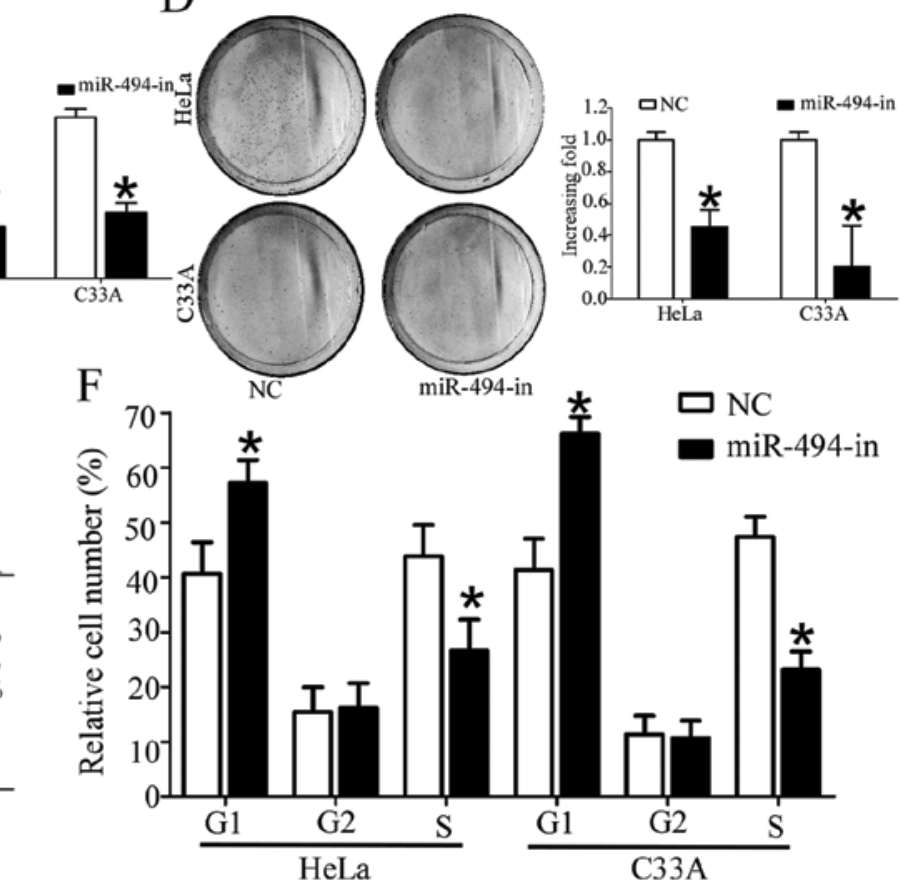

Figure 3. Effects of miR-494 inhibition on cell proliferation and cell cycle of cervical cancer cells. (A) Expression of miR-494 in HeLa and C33A cells transfected with miR-494 inhibitor. (B) Effects of miR-494 inhibition on the cell viability of HeLa and C33A cervical cancer cells. (C) Effects of miR-494 inhibition on soft-agar colony formation ability of HeLa and C33A cervical cancer cells. (D) Effects of miR-494 inhibition on colony formation ability of HeLa and C33A cervical cancer cells. (E and F) Effects of miR-494 on cell cycle of HeLa and C33A cervical cancer cells. Three independent experiments were performed in duplicate. Data are presented as mean \pm SD. Two-tailed Student's t-test was used to analyze the significant differences. "P $<0.05$.

in HeLa and C33A (Fig. 3A). After confirming the efficiency of miR-494 inhibitors, we determined the effects of miR-494 on cell viability using an MTT assay. Cervical cancer cells transfected with miR-494 inhibitors showed a significant decrease in cell viability as compared with the normal control (Fig. 3B). We determined the effect of miR-494 on cell proliferation using colony formation and soft agar colony formation assays. As shown in Fig. 4C and D, inhibition of miR-494 significantly decreased the growth rate of the two cervical cell lines as compared with the normal control (Fig. 4C and D). Taken together, these results indicated that the downregulation of miR-494 suppressed the proliferation of cervical cancer cells.

Effect of miR-494 on cell cycle in vitro. As miR-494 significantly affects cell proliferation in HeLa and C33A cells, we hypothesized that miR-494 functions by affecting the cell cycle of cervical cancer cells. Thus, we investigated the effect of miR-494 on the cell cycle by flow cytometry. The results revealed that overexpression of miR-494 inhibitors markedly increased the number of cells in G1 peak and decreased those in the $\mathrm{S}$ peak (Fig. 3E and F). Taken together, these results indicated the inhibition of miR-494 suppressed the proliferation of cervical cancer cells by inducing cell cycle arrest.

Inhibition of miR-494 increases cell cycle inhibitors $p 21^{\text {Cipl }}$ and decreases cell cycle regulator cyclin D1. As overexpression of miR-494 inhibitors appears tobe closely linked to the proliferation of cervical cancer cells, we further investigated whether the $\mathrm{CDK}$ inhibitor $\mathrm{p} 21^{\mathrm{Cip} 1}$ or the $\mathrm{CDK}$ regulator cyclin D1 could be regulated by miR-494. RT-qPCR and western blot analysis revealed that $\mathrm{p} 21^{\mathrm{Cip} 1}$ was upregulated, whereas cyclin D1 was downregulated in cervical cells transfected with miR-494 inhibitors compared with cells transfected with the normal control (Fig. 4A and B). Taken together, these results supported our hypothesis that miR-494 has a critical role in the growth of cervical cancer cells. 
A

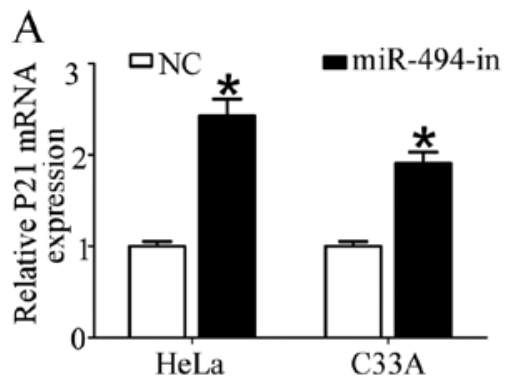

C
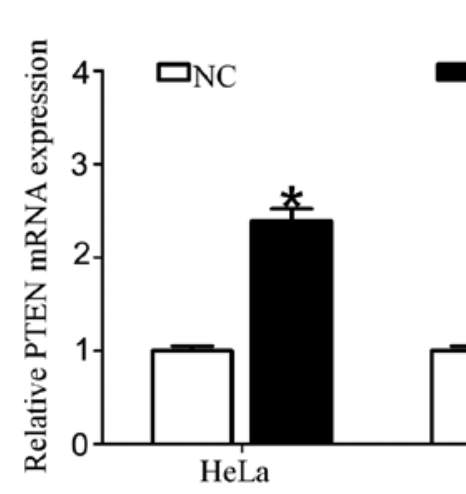

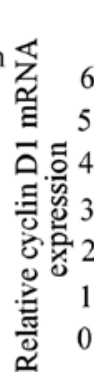

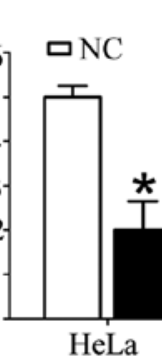

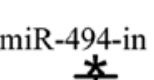

$\mathrm{E}$

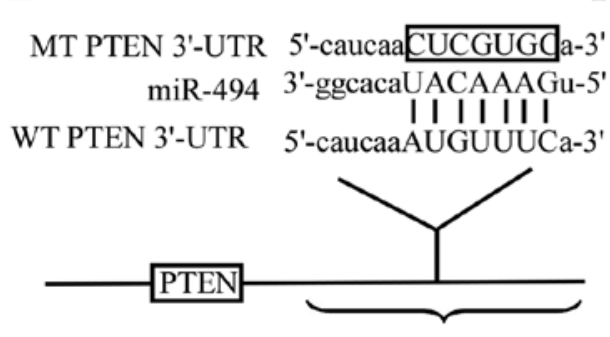

3'-UTR

B

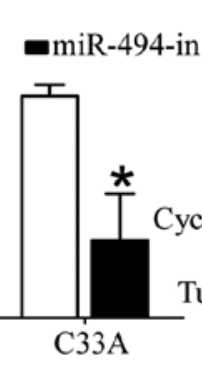

D

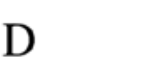

B
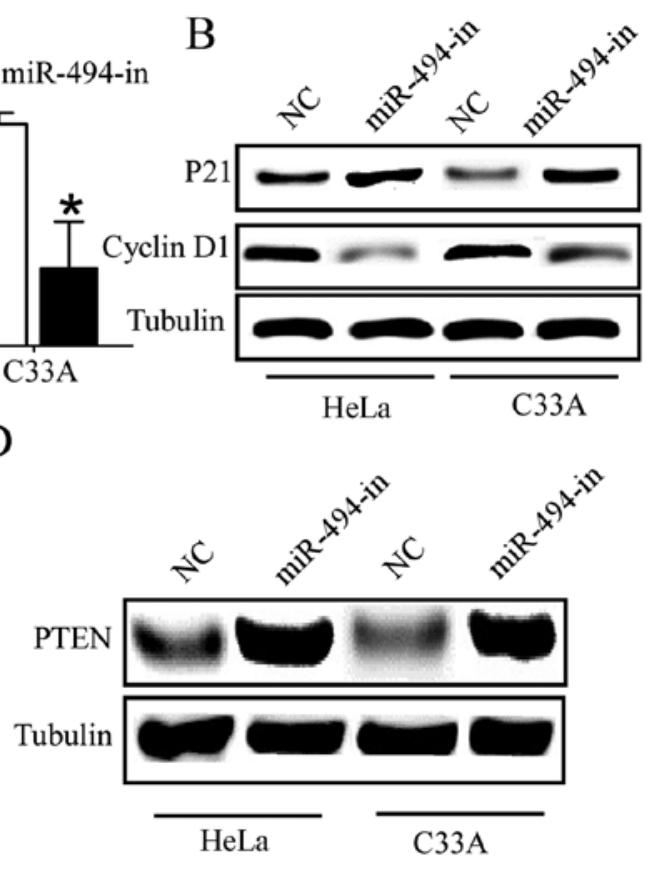

\section{.}

$\mathrm{F}$

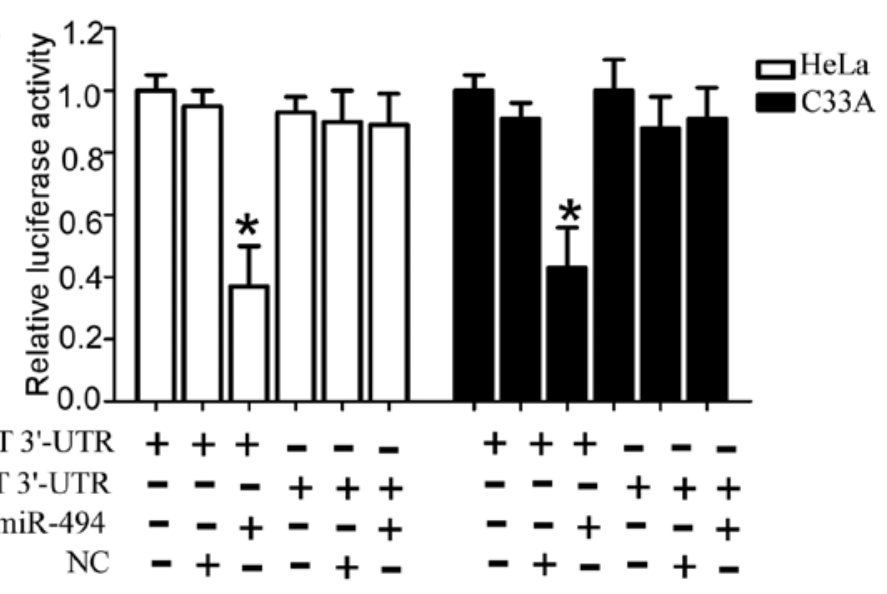

Figure 4. PTEN is the direct target of miR-494. (A) Expression of p21 ${ }^{\text {Clipl }}$ and cyclin D1 in HeLa and C33A cells following transfection with miR-494 or NC by RT-qPCR. (B) Expression of p21 Clip1 and cyclin D1 in HeLa and C33A cells following transfection with miR-494 or NC by western blot analysis. Tubulin was used as a loading control. (C) Expression of PTEN in HeLa and C33A cells after transfection with miR-494 or NC by RT-qPCR. (D) Expression of PTEN in HeLa and C33A cells after transfection with miR-494 or NC by western blot analysis. Tubulin was used as a loading control. (E) miR-494 and its putative binding sequence in the 3'-UTR of PTEN, and the diagram of the luciferase reporter plasmids with WT and MT PTEN 3'-UTR. (F) Luciferase assay on HeLa and C33A cells transfected with the plasmids containing WT or MT PTEN 3'-UTR and miR-494 inhibitor oligonucleotides, as indicated. Three independent experiments were performed in duplicate. Data are expressed as means \pm SD. Two-tailed Student's t-test was used to analyze the significant differences. ${ }^{\prime} \mathrm{P}<0.05$. PTEN, phosphatase and tensin homolog deleted on chromosome 10; NC, negative control, WT, wild-type; MT, mutant; 3'-UTR, 3'-untranslated region.

PTEN is the direct target of miR-494 in cervical cancer cells. It has been proven that PTEN was the direct target of miR-494 in multiple solid tumors (24), and loss of protein expression of PTEN was involved in the pathogenesis, proliferation and metastasis of cervical cancer $(25,26)$. Considering the tissue-specific and developmental stage-specific manner of miRNA, we investigated the relationship between PTEN and miR-494 in cervical cancer. In order to confirm PTEN is the target gene for miR-494 in cervical cancer cells, RT-qPCR and western blotting was used to detect the expression of PTEN in HeLa and C33A. As expected, the expression of PTEN at the mRNA and protein level was significantly upregulated in cervical cancer cells transfected with miR-494 inhibitors (Fig. 4C and D). Our previous results demonstrated the mRNA of PTEN was inversely correlated with miR-494 expression (Fig. 1D). Taken together, these results suggested that PTEN was the potential target gene of miR-494 in cervical cancer cell lines and tissues.

Then we performed the luciferase reporter assay to further verify whether miR-494 directly targeted the 3'-UTR of PTEN in cervical cancer cells. The target sequence of wild-type PTEN 3'-UTR (WT 3'-UTR) or the mutant PTEN 3'-UTR (MT 3'-UTR) was cloned into a luciferase reporter vector (Fig. 4E). As shown in Fig. 4F, transfection of miR-494 consistently suppressed the luciferase activity of PTEN WT3'UTR luciferase reporter plasmids in HeLa and C33A cells, whereas point mutations in the miR-494-binding seed region of the PTEN abrogated the repressive effect of miR-494. Taken together, the data suggested that PTEN was a genuine target of miR-494. 


\section{Discussion}

In the present study, miR-494 expression was significantly upregulated in human cervical cancer cell lines and tissues. miR-494 upregulation was also significantly associated with PTEN downregulation, adverse clinicopathological characteristics, poor overall and progression-free survival, and poor prognosis. In addition, inhibition of miR-494 expression induced cell cycle arrest in G1 stage and inhibited cell proliferation and cell growth in cervical cancer cell lines. Additional in vitro studies showed that PTEN was the direct target of miR-494 in cervical cancer cells. Results of the present study show that miR-494 may have an essential role in the carcinogenesis and progression of cervical cancer.

Several miRNAs have been identified as candidate components of oncogene and tumor suppressor networks in cervical cancer, and these miRNAs and their targets play critical roles in the carcinogenesis and progression of cervical cancer. For example, miR-135a/SIAH1/ $\beta$-catenin signaling functions as an oncogene in the transformation and progression of cervical cancer (27). Similarly, miR-31, miR-155 and miR-1246 are found to promote cervical cancer cell proliferation and function as oncomiRs in cervical cancer (28-30). However, miR-507, miR-99a and miR-99b act as tumor suppressors in cervical cancer and inhibit cervical cancer cell proliferation and cell growth $(31,32)$. Classical tumor suppressor miR-101 induced cell cycle arrest by targeting Fos (33). However the role, mechanism and clinical significance of miR-494 in cervical cancer have not been further reported, since whether miR-494 is an oncogenic or tumor suppressor miRNA remains to be determined.

Accumulating evidence suggested that the functions of miR-494 in cancer development are complicated. Upregulation of miR-494 has been proven to be associated with promotion in cell proliferation and cell growth in H460 lung and breast cancer cells, colorectal cancer, hepatocellular carcinoma and transformed bronchial epithelial cells $(18,24,34-36)$. However, miR-494 functions as a tumor suppressor and induces cell cycle arrest in lung, gastric and prostate cancer, and cholangiocarcinoma $(17,37,38)$. Different tumor microenvironments, cellular contexts, tissue specificity and molecules which miR-494 targeted account for this discrepancy. As the effect of miR-494 in cervical cancer was far from defined, the present study aimed to investigate the potential biological function of miR-494 in cervical cancers. Our results demonstrate that suppression of miR-494 significantly inhibited the cell proliferation and cell growth of HeLa and C33A cells by induction of cell cycle arrest. More importantly, miR-494 was significantly correlated with adverse clinicopathological features, poor survival and prognosis of cervical cancer patients. All these results suggest that miR-494 may function as an oncogenic miRNA in the initiation and progression of cervical cancer. Of note, the present study further supported the hypothesis that various functions of miRNA, including miR-494, in different types of cancer were dependent on the cancer type and cellular context (28).

To address the molecular mechanism involved in miR-494-mediated changes of biological properties, PTEN was selected for further study. The PTEN gene, known as mutated in multiple advanced cancer 1 (MMAC1), is a classic tumor-suppressor gene located at chromosome 10q23.31 (39).
The PTEN protein is principally involved in the homeostatic maintenance of PI3K/Akt signaling (40). PTEN/PI3K/Akt is highly involved in carcinogenesis and associated with EMT (41), and cell cycle arrest $(42,43)$. The PI3K/Akt signaling pathway is involved in tumor cell proliferation during the development of cervical cancer, and downstream effectors of PI3K/Akt signaling are promising targets for cervical cancer therapy (4). Gene expression profiling also suggests that the PI3K/Akt pathway is a therapeutic target in cervical cancer (6). More importantly, it has been confirmed that PTEN, which counteracts PI3K/Akt activity, is involved in various aspects of cancer development, such as inhibition of cell proliferation, apoptosis, migration and invasion $(8,44,45)$. In particular, PTEN expression intensity is lower in cervical cancer than benign cervical samples (46) and a decreased expression of PTEN was found in invasive cervical cancers (47). From the previous study, we concluded that the PTEN/PI3K/Akt signaling pathway is important in the carcinogenesis and development of cervical cancer, thus identifying the molecules regulating PTEN/PI3K/Akt may be an attractive strategy for the underlying mechanism of cervical carcinogenesis. In the present study, the results supported the hypothesis as, miR-494 was involved in the modulation of PTEN expression in cervical cancer. First of all, miR-494 was inversely correlated with PTEN expression in cervical cancer tissues. Secondly, the mRNA and protein levels of PTEN were significantly upregulated after knockdown of miR-494 expression in cervical cancer cell lines. In addition, luciferase analyses indicated PTEN was the direct target of miR-494 in cervical cancer cells. Taken together, we have demonstrated miR-494 could directly regulate PTEN expression by targeting its mRNA 3'-UTR. Thus, previous findings and our results suggest that, miR-494 functions as an oncogenic miRNA and PTEN/PI3K/Akt regulator in cervical cancer. More specific studies are required to further elucidate the relationship between miR-494 and PI3K/Akt and more specific mechanisms that miR-494 regulated the expression of PTEN.

In summary, to the best of our knowledge, the present study identified for the first time the correlation between miR-494-mediated cervical cancer cell proliferation and downregulation of PTEN. Our findings reveal a crucial role for miR-494 in regulating cell cycle checkpoints and cervical cancer cell proliferation. Understanding the precise role played by miR-494 in inducing tumor cell proliferation may increase our understanding of the biology of cervical cancer and inhibition of miR-494 may be a novel therapeutic strategy in the treatment of cervical cancer.

\section{Acknowledgements}

The authors would like to thank the local doctors and the patients who participated in the present study.

\section{References}

1. Siegel R, Ma J, Zou Z and Jemal A: Cancer statistics, 2014. CA Cancer J Clin 64: 9-29, 2014.

2. Castellsagué X, Díaz M, de Sanjosé S, et al: Worldwide human papillomavirus etiology of cervical adenocarcinoma and its cofactors: implications for screening and prevention. J Natl Cancer Inst 98: 303-315, 2006. 
3. Hildesheim A and Wang SS: Host and viral genetics and risk of cervical cancer: a review. Virus Res 89: 229-240, 2002.

4. Du CX and Wang Y: Expression of P-Akt, NFkappaB and their correlation with human papillomavirus infection in cervical carcinoma. Eur J Gynaecol Oncol 33: 274-277, 2012.

5. Castellsagué X: Natural history and epidemiology of HPV infection and cervical cancer. Gynecol Oncol 110 (Suppl 2): S4-S7, 2008

6. Schwarz JK, Payton JE, Rashmi R, et al: Pathway-specific analysis of gene expression data identifies the PI3K/Akt pathway as a novel therapeutic target in cervical cancer. Clin Cancer Res 18: 1464-1471, 2012.

7. Molinari $\mathrm{F}$ and Frattini M: Functions and regulation of the PTEN gene in colorectal cancer. Front Oncol 3: 326, 2014.

8. Zhang S and Yu D: PI(3)king apart PTEN's role in cancer. Clin Cancer Res 16: 4325-4330, 2010.

9. Qi Q, Ling Y, Zhu M, et al: Promoter region methylation and loss of protein expression of PTEN and significance in cervical cancer. Biomed Rep 2: 653-658, 2014.

10. Inui M, Martello G and Piccolo S: MicroRNA control of signal transduction. Nat Rev Mol Cell Biol 11: 252-263, 2010.

11. Ventura A and Jacks T: MicroRNAs and cancer: short RNAs go a long way. Cell 136: 586-591, 2009.

12. Bartels CL and Tsongalis GJ: MicroRNAs: novel biomarkers for human cancer. Clin Chem 55: 623-631, 2009.

13. Zhao JJ, Yang J, Lin J, et al: Identification of miRNAs associated with tumorigenesis of retinoblastoma by miRNA microarray analysis. Childs Nerv Syst 25: 13-20, 2009

14. Kwak SY, Yang JS, Kim BY, Bae IH and Han YH: Ionizing radiation-inducible miR-494 promotes glioma cell invasion through EGFR stabilization by targeting p190B rhoGAP. Biochim Biophys Acta 1843: 508-516, 2014.

15. Yamanaka S, Campbell NR, An F, et al: Coordinated effects of microRNA-494 induce $\mathrm{G}_{2} / \mathrm{M}$ arrest in human cholangiocarcinoma. Cell Cycle 11: 2729-2738, 2012.

16. Li X, Luo F, Li Q, et al: Identification of new aberrantly expressed miRNAs in intestinal-type gastric cancer and its clinical significance. Oncol Rep 26: 1431-1439, 2011

17. Olaru AV, Ghiaur G, Yamanaka S, et al: MicroRNA down-regulated in human cholangiocarcinoma control cell cycle through multiple targets involved in the G1/S checkpoint. Hepatology 54 2089-2098, 2011.

18. Sun HB, Chen X, Ji H, et al: miR494 is an independent prognostic factor and promotes cell migration and invasion in colorectal cancer by directly targeting PTEN. Int J Oncol 45: 2486-2494, 2014.

19. Lui WO, Pourmand N, Patterson BK and Fire A: Patterns of known and novel small RNAs in human cervical cancer. Cancer Res 67: 6031-6043, 2007.

20. Lee JW, Choi CH, Choi JJ, et al: Altered microRNA expression in cervical carcinomas. Clin Cancer Res 14: 2535-2542, 2008.

21. FIGO Committee on Gynecologic Oncology: FIGO staging for carcinoma of the vulva, cervix, and corpus uteri. Int J Gynaecol Obstet 125: 97-98, 2014

22. Hou T, Ou J, Zhao X, Huang X, Huang Y and Zhang Y: MicroRNA-196a promotes cervical cancer proliferation through the regulation of FOXO1 and p27 ${ }^{\mathrm{Kip} 1}$. Br J Cancer 110: 1260-1268, 2014.

23. El-Mansi MT and Williams AR: Evaluation of PTEN expression in cervical adenocarcinoma by tissue microarray. Int $\mathrm{J}$ Gynecol Cancer 16: 1254-1260, 2006.

24. Liu Y, Lai L, Chen Q, et al: MicroRNA-494 is required for the accumulation and functions of tumor-expanded myeloid-derived suppressor cells via targeting of PTEN. J Immunol 188 : 5500-5510, 2012

25. Zhang GW, Lin JH, Qian JP and Zhou J: Identification of risk and prognostic factors for patients with clonorchiasis-associated intrahepatic cholangiocarcinoma. Ann Surg Oncol 21: 3628-3637, 2014.

26. Lu D, Qian J, Yin X, Xiao Q, Wang C and Zeng Y: Expression of PTEN and survivin in cervical cancer: promising biological markers for early diagnosis and prognostic evaluation. Br J Biomed Sci 69: 143-146, 2012.
27. Leung CO, Deng W, Ye TM, et al: miR-135a leads to cervical cancer cell transformation through regulation of $\beta$-catenin via a SIAH1-dependent ubiquitin proteosomal pathway. Carcinogenesis 35: 1931-1940, 2014

28. Wang N, Zhou Y, Zheng L and Li H: MiR-31 is an independent prognostic factor and functions as an oncomir in cervical cancer via targeting ARID1A. Gynecol Oncol 134: 129-137, 2014.

29. Lao G, Liu P, Wu Q, et al: Mir-155 promotes cervical cancer cell proliferation through suppression of its target gene LKB1. Tumour Biol 35: 11933-11938, 2014.

30. Chen J, Yao D, Zhao S, et al: MiR-1246 promotes SiHa cervical cancer cell proliferation, invasion, and migration through suppression of its target gene thrombospondin 2. Arch Gynecol Obstet 290: 725-732, 2014.

31. Wen SY, Lin Y, Yu YQ, et al: miR-506 acts as a tumor suppressor by directly targeting the hedgehog pathway transcription factor Gli3 in human cervical cancer. Oncogene: Mar 10, 2014 (Epub ahead of print). doi: 10.1038/onc.2014.9.

32. Wang L, Chang L, Li Z, et al: miR-99a and -99b inhibit cervical cancer cell proliferation and invasion by targeting mTOR signaling pathway. Med Oncol 31: 934, 2014.

33. Liang X, Liu Y, Zeng L, et al: miR-101 inhibits the G1-to-S phase transition of cervical cancer cells by targeting Fos. Int J Gynecol Cancer 24: 1165-1172, 2014

34. Liu L, Jiang Y, Zhang H, Greenlee AR and Han Z Overexpressed miR-494 down-regulates PTEN gene expression in cells transformed by anti-benzo(a)pyrene-trans-7,8-dihydrodiol-9,10-epoxide. Life Sci 86: 192-198, 2010.

35. Lim L, Balakrishnan A, Huskey N, et al: MicroRNA-494 within an oncogenic microRNA megacluster regulates $G_{1} / S$ transition in liver tumorigenesis through suppression of mutated in colorectal cancer. Hepatology 59: 202-215, 2014.

36. Romano G, Acunzo M, Garofalo M, et al: MiR-494 is regulated by ERK1/2 and modulates TRAIL-induced apoptosis in non-small-cell lung cancer through BIM down-regulation. Proc Natl Acad Sci USA 109: 16570-16575, 2012.

37. Ohdaira H, Sekiguchi M, Miyata K and Yoshida K: MicroRNA-494 suppresses cell proliferation and induces senescence in A549 lung cancer cells. Cell Prolif 45: 32-38, 2012.

38. Shen PF, Chen XQ, Liao YC, et al: MicroRNA-494-3p targets CXCR4 to suppress the proliferation, invasion, and migration of prostate cancer. Prostate 74: 756-767, 2014.

39. Correia NC, Gírio A, Antunes I, Martins LR and Barata JT: The multiple layers of non-genetic regulation of PTEN tumour suppressor activity. Eur J Cancer 50: 216-225, 2014.

40. Hafsi S, Pezzino FM, Candido S, et al: Gene alterations in the PI3K/PTEN/AKT pathway as a mechanism of drug-resistance (Review). Int J Oncol 40: 639-644, 2012.

41. Mulholland DJ, Kobayashi N, Ruscetti M, et al: Pten loss and RAS/MAPK activation cooperate to promote EMT and metastasis initiated from prostate cancer stem/progenitor cells. Cancer Res 72: 1878-1889, 2012

42. Choi BH, Pagano M and Dai W: Plk1 protein phosphorylates phosphatase and tensin homolog (PTEN) and regulates its mitotic activity during the cell cycle. J Biol Chem 289: 14066-14074, 2014.

43. Vogt PK, Gymnopoulos M and Hart JR: PI 3-kinase and cancer: changing accents. Curr Opin Genet Dev 19: 12-17, 2009.

44. Stewart AL, Mhashilkar AM, Yang XH, et al: PI3 kinase blockade by Ad-PTEN inhibits invasion and induces apoptosis in RGP and metastatic melanoma cells. Mol Med 8: 451-461, 2002.

45. Tamura M, Gu J, Matsumoto K, Aota S, Parsons R and Yamada KM: Inhibition of cell migration, spreading, and focal adhesions by tumor suppressor PTEN. Science 280: 1614-1617, 1998.

46. Loures LF, Cândido EB, Vidigal PV, Seabra MA, Marco LA and Silva-Filho AL: PTEN expression in patients with carcinoma of the cervix and its association with p53, Ki-67 and CD31. Rev Bras Ginecol Obstet 36: 205-210, 2014.

47. Vázquez-Ulloa E, Lizano M, Aviléss-Salas A, Alfaro-Moreno E and Contreras-Paredes A: Abnormal distribution of hDlg and PTEN in premalignant lesions and invasive cervical cancer. Gynecol Oncol 122: 663-668, 2011. 